\title{
Ressources en ligne
}

Une sélection de ressources en ligne sur l'éducation en Asie

\section{Marion Latour}

\section{OpenEdition}

\section{Journals}

Édition électronique

URL : http://journals.openedition.org/ries/376

DOI : $10.4000 /$ ries.376

ISSN : 2261-4265

Éditeur

Centre international d'études pédagogiques

Édition imprimée

Date de publication : 1 septembre 2008

Pagination : 10-12

ISBN : 978-2-85420-573-2

ISSN : $1254-4590$

Référence électronique

Marion Latour, «Ressources en ligne », Revue internationale d'éducation de Sèvres [En ligne], 48 | septembre 2008, mis en ligne le 27 juin 2011, consulté le 26 avril 2021. URL : http:// journals.openedition.org/ries/376 ; DOI : https://doi.org/10.4000/ries.376

Ce document a été généré automatiquement le 26 avril 2021.

(c) Tous droits réservés 


\title{
Ressources en ligne
}

Une sélection de ressources en ligne sur l'éducation en Asie $^{1}$

\author{
Marion Latour
}

1 Cet article s'inscrit dans la continuité de celui du $\mathrm{n}^{\circ} 45$ de septembre 2007 sur l'Amérique latine et les Caraïbes, et de celui du n ${ }^{\circ} 46$ de décembre 2007 sur la politique de l'Union européenne en matière d'éducation et de formation. Les ressources présentées ici, d'accès libre et gratuit, émanent d'institutions mondiales ou régionales, qui proposent des ressources seulement en anglais, à l'exception de l'Association internationale des universités (AIU), de l'Institut international de planification de l'éducation (IIPE) et de l'Institut de statistique de l'Unesco.

2 Sitographie arrêtée le 13 juin 2008.

\section{Institutions internationales}

\section{Association internationale des universités (AIU)}

3 Affiliée à l'Unesco, cette organisation regroupe les universités de près de centre cinquante pays afin de croiser réflexions et actions sur leurs préoccupations communes. Ses thématiques de travail concernent en particulier l'accès à l'enseignement supérieur, l'éducation pour tous, l'internationalisation et le dialogue interculturel. L'AIU tient à jour plusieurs bases de données dont celle sur les systèmes d'enseignement (pour tout niveau mais plus particulièrement le supérieur) de quelque 180 pays et territoires. Une version en français de cette base de données, éditée en 2005, est téléchargeable sur le site. L'information est également disponible en anglais pour l'année scolaire 2005/2006; l'interrogation se fait par pays: http:// www.unesco.org/iau/fre/index.html

\section{Banque mondiale}

Organisme international apportant un appui technique et financier aux pays en développement, la Banque mondiale soutient des projets éducatifs dans seize pays 
d'Asie orientale et du Pacifique. Dans la rubrique consacrée à cette zone et à la thématique éducative sont rassemblés les projets et programmes menés par la Banque, les publications - qu'ils s'agissent de rapports régionaux ou nationaux - ainsi que des données statistiques. Il est également possible d'accéder à des données en éducation par les profils par pays, qui regroupent des synthèses sur la situation éducative, des indicateurs nationaux et internationaux et différentes statistiques. Enfin, la banque de données des projets en éducation menés par la Banque entre 1996 et 2007 permet une interrogation par pays et par thèmes : http://www.banquemondiale.org/

\section{Bureau international d'éducation (BIE)}

5 Le BIE est un centre de l'Unesco spécialisé dans le développement des contenus éducatifs, qui œuvre en particulier au partage des expériences et des compétences sur le développement curriculaire et à la coopération régionale et internationale. Il propose sur son site un espace "Dossiers pays", dans lequel on entre par régions (AsiePacifique) ou par pays. Y sont regroupés des données sur les systèmes éducatifs (extraits de la base de données mondiales en éducation également éditée par le BIE, dont la version en ligne date d'octobre 2006), sur les curricula ainsi que des liens vers des sources officielles d'information sur l'éducation:

http://www.ibe.unesco.org/French/asia_fr.htm

\section{Institut international de planification de l'éducation (IIPE)}

Ce centre de formation et de recherche de l'Unesco est spécialisé en planification et gestion de l'éducation. Il propose un portail sur les plans et politiques d'éducation des États membres de l'Organisation, Planipolis. L'interface de recherche simple permet une interrogation par région ou pays, mots clés et type de document, notamment les plans nationaux d'éducation - dont ceux pour l'éducation pour tous -, les politiques nationales d'éducation et les rapports sur les objectifs du millénaire pour le développement. Lorsqu'on interroge par pays, plusieurs liens sont proposés: en particulier la fiche des indicateurs clés sur tous les niveaux du système éducatif, établie par l'Institut statistique de l'Unesco (ISU) ; les rapports retraçant les activités de l'IIPE dans le pays ; les références bibliographiques de la base de données de l'IIPE, avec accès au document final lorsque cela est possible. Un formulaire de recherche avancée propose une recherche multicritères : http://www.unesco.org/iiep/fre/indexfr.htm

\section{Institut de statistique de l'Unesco (ISU)}

7 Bureau de statistique de l'Unesco, l'ISU a pour mission de collecter, analyser et diffuser les données statistiques dans les domaines d'intervention de l'Unesco, au profit de la communauté internationale. Il publie des résumés et des études statistiques, des rapports de recherche ainsi que des manuels méthodologiques et des classifications internationales. Parmi ses publications clés figure le Recueil de données mondiales sur l'éducation, qui permet une lecture comparée des statistiques internationales. Le site propose des statistiques par pays pour chacun des niveaux d'enseignement: http:// www.uis.unesco.org/ 


\section{Organisations régionales}

\section{Banque asiatique de développement (BAD)}

8 Regroupant soixante-sept pays membres dont quarante-huit de la zone Asie, cet organisme financier international est spécialisé dans la lutte contre la pauvreté, notamment à travers des actions en éducation auxquelles il consacre une rubrique sur son site. Outre les documents des projets menés pas la Banque, on trouve des outils tels que des analyses économiques et financières des systèmes et projets éducatifs ainsi que des exemples de bonnes pratiques (technologies de l'information et de la communication, approches sectorielles, études de cas pays). Une base de données en éducation regroupe plus d'une centaine de documents en ligne depuis 1992. Il est également possible d'accéder à un ensemble de ressources (projets, publications et actualités), tous secteurs confondus, par zones géographiques et par pays: http:// www.adb.org/

\section{Asian Development Bank Institute (ADBI)}

9 Créé en 1997 à la demande des États membres des pays en développement, l'Institut répond à deux besoins, la formation au management du développement et l'échange d'idées sur les sujets de politique, au travers de la recherche appliquée, de séminaires et d'un certain nombre d'initiatives sur le développement des capacités. Les publications reprennent les actes des conférences et les différentes productions des chercheurs. Une centaine de documents traitent de l'éducation : http://www.adbi.org/

\section{Southeast Asian Ministers of Education Organization (SEAMEO)}

10 Fondée en 1965, cette organisation a pour vocation de promouvoir l'éducation, la science et la culture au sein des onze pays membres situés en Asie du Sud-est. Cette coopération régionale se traduit notamment par la mise en œuvre de partenariats, de réseaux (quinze centres spécialisés dans huit États membres) et d'un forum pour les experts et responsables des politiques éducatives. Outre quelques numéros de revues spécialisées en éducation disponibles sur le site, une bibliothèque en ligne propose notamment l'accès à une base de données bibliographiques (avec accès au document quand cela est possible), des actes de colloques, des projets de recherche et divers publications témoignant des activités de la SEAMO. Une rubrique rassemble des statistiques en éducation émanant pour l'essentiel des ministères des pays membres : http://www.seameo.org

\section{Unesco Bangkok}

11 Inauguré en 1961, le Bureau de Bangkok était initialement le Bureau régional de l'Unesco de la zone Asie pour l'éducation primaire et l'enseignement obligatoire. Puis il s'est ouvert aux pays de la région Pacifique - regroupant actuellement quarante-quatre États membres - et à tous les niveaux du système éducatif, avant d'élargir ses activités à la culture et aux sciences humaines et sociales. Unesco Bangkok propose une bibliothèque en ligne qui donne accès aux informations sur cette zone géographique, 
toutes thématiques confondues, notamment un répertoire commenté de liens vers des profils par pays. Le Bureau consacré à l'éducation dans cette zone se décline en trois départements qui s'intéressent à l'éducation pour tous, aux innovations éducatives pour le développement et aux politiques et réformes de l'éducation. Le site propose également des informations sur la vingtaine de programmes en jeu dans la région, enseignement primaire, enseignement secondaire, changements curriculaires, ou égalité des genres, pour n'en citer que quelques-uns : http://www.unescobkk.org/

\section{NOTES}

1. Pour faciliter la lecture, seule la racine des liens internet est mentionnée dans cet article. Pour y accéder dans leur intégralité, il suffit d'aller sur la page d'accueil du CIEP (http://www.ciep.fr/), d'ouvrir la rubrique Ressources documentaires, puis Produits documentaires pour accéder aux Sitographies.

INDEX

Mots-clés : coopération en éducation, organisation internationale, système éducatif Index géographique : Asie

\section{AUTEUR}

\section{MARION LATOUR}

Documentaliste, CIEP 